\title{
Interspecific Hybridization in Genus Abelmoschus Medikus
}

\author{
Kattula Nagaraju ${ }^{1 *}$, M. Pitchaimuthu ${ }^{1}$, A.T. Sadashiva ${ }^{1}$, \\ E.S. Rao ${ }^{1}$, A. Rekha ${ }^{3}$ and R. Venugopalan ${ }^{2}$ \\ ${ }^{1}$ Division of Vegetable Crops, ICAR-IIHR, Bengaluru, Karnataka-560089, India \\ ${ }^{2}$ Statistics Lab, ICAR-IIHR, Bengaluru, Karnataka-560089, India \\ ${ }^{3}$ Cytology Lab, Division of Fruit Crops, ICAR- IIHR, Karnataka-560089, India \\ *Corresponding author
}

\section{A B S T R A C T}

\begin{abstract}
Interspecific hybridization in okra is of immense value to exploit the diversity of species in the genus Abelmsochus for developing male sterile lines and/or advance breeding lines possessing resistance to YVMV and ELCV diseases. The present investigation was carried out during the year 2015-16 to study the pattern of interspecific hybridization in genus Ablemoschus involving ten wild species viz., A. angulosus var. angulosus, A. angulosus var. grandiflorus, A. caillei, A. ficulneus, A. manihot ssp. tetraphyllus, A. moschatus, A. tetraphyllus var. pungense, A. tetraphyllus var. tetraphyllus, A. tuberculatus and new taxa IC 0433556 with eight genotypes of cultivated species (Abelmoschus esculentus). Hand pollination was undertaken; fruit set, seed set, seed germination and pollen viability per cent were calculated. From the present investigation, it was found that the cultivated A. esculentus genotypes were compatible as male parents with A. caillei, A. manihot ssp. tetraphyllus and A. tetraphyllus var. tetraphyllus, A. tuberculatus. Interestingly, there was no compatibility when A. tetraphyllus var. pungense and new taxa IC 0433556 crossed with cultivated okra genotypes (IIHR-285 and 10-11-594). However, there was limited fruit set observed in A. angulosus var. angulosus, A. angulosus var. grandiflorus, A. moschatus and A. ficulneus. The $\mathrm{F}_{1}$ seeds thus obtained were failed to germinate due to non-viable seeds. The significant difference $(\mathrm{p}<0.01)$ was found among the interspecific hybrids for pollen viability per cent. Germination per cent was higher in wild and cultivated parents than that of interspecific hybrids. Crossability index per cent was ranged from zero to hundred per cent (0-100\%) among the selected cross combinations.
\end{abstract}

\section{Keywords}

Abelmoschus, Okra, Interspecific

hybridization, Wild species, Crossability, seed set, Fruit set, Pollen viability

Article Info

Accepted:

04 July 2019

Available Online:

10 August 2019

\section{Introduction}

Okra [Abelmoschus esculentus (L.) Moench] is an economical and traditional vegetable belongs to Malvaceae family which is widely grown in both the tropical and sub-tropical regions of the world (Eshiet and Brisibe, 2015). It has both culinary and medicinal properties and hence it has a potential role in healthy diet in human life (Gemede et al.,
2014, Ohr, 2004 and Gosslau and Chen, 2004). In okra production, India occupies top position in the world. Its cultivated area, production and productivity are 0.51 million ha, 6.2 million tones and 12.2 t/ha respectively (Indiastat, 2018). The major causes for low productivity in okra are limited breeding efforts to develop high yielding cultivars and lack of resistant sources to biotic and abiotic stresses. Major biotic factors causing yield 
losses in okra are YVMV, ELCV, fusarium wilt and powdery mildew, shoot and fruit borer, hoppers, aphids and jassids. There is limited genetic variability and limited resistant sources availability in cultivated Abelmoschus esculentus are the major lacunae to overcome these diseases and pests.

In okra, only marginal improvement in yield was achieved through traditional hybridization combined with selection. Though okra is often cross pollinated crop, high level of heterosis has been reported for yield and its components. But, the commercial exploitation of this situation is realised only if the hybrid seeds produced economically i.e., by utilizing male sterility system which will circumvent time consuming and laborious hand emasculation.

Further, presence of convincible reports on heterosis for yield in okra, developing cytoplasmic male sterility (CMS) system in okra using the potential wild donors can facilitate the production of hybrid varieties with higher yield, increased resistance to biotic and abiotic stresses. The presence of male sterility in agricultural and horticultural crops and its importance in developing $F_{1}$ hybrids has lead to superabundant research work pertaining to manipulations in extranuclear genome (Yarrow, 1993).

The introgression of nucleus of one species into the cytoplasmic background of the alien species through backcrossing has been commercially exploited in many crops. A number of different male sterility systems are now available in vegetable crops like cole crops, brinjal, chilli, capsicum and commercial crops like cotton, sunflower and safflower etc. In cole crops, male sterility system was obtained by alloplasmic substitution of the cytoplasm from other species of genus Brassica and its wild leatives (Yamagishi and Bhatt, 2014).
Apart from creation of male sterility, inter specific hybridization enables transfer of specific disease and pest resistance traits from wild and/or related species to cultivated species. Some of the wild okra species are reported to have desirable genes like disease and pest resistance (Singh et al., 2007). For the first time, male sterility in okra has been induced by gamma radiation and it is reported to be conditioned by single recessive gene $\left(m s_{1}\right)$ (Dutta, 1976). In the present study, by employing interspecific hybridization, attempt was done for finding male sterility-inducing cytoplasm among wild species of the genus Abelmoschus with a view to develop and exploit CGMS system owing to the problems associated with the currently used GMS (existence of $50 \%$ pollen shedders in seed parent block and thus need to rogue out male fertile plants) in the development of commercial okra hybrids (Pitchaimuthu et al., 2012).

The genus Abelmsochus in India consists of about 12 species, out of which three are cultivated and the rest are wild species (Joseph et al., 2017). Differential ploidy level and often genotype specific variation in chromosome number has been noticed in okra as well as some of its wild relatives (Keisham et al., 2014). The crop is facing challenges of YVMV and ELCV which require search for resistance in the wild related taxa and their incorporation in cultivated okra. Attempts to develop hybrids of okra with different wild species were ruined due to sterility of the $F_{1}$ hybrid. The Crosses between cultivated okra genotypes and wild okra species viz., $A$. angulosus var. grandiflorus, A. caillei, A. tuberculatus, A. tetraphyllus and $A$. mizonagensis sp. nov. (Mizoram), $A$. moschatus subsp. rugosus and Guinean okra with A. esculentus, A. angulosus var. grandiflorus and A. mizonagensis sp. nov. (Mizoram) were raised but could not be advanced to next generation as the 
interspecific hybrid failed to produce viable seeds upon selfing and back cross.

In general, okra wild species had many desirable traits like dark green colour, high mucilage, extended bearing, perennation tendency, high branching, reduced fruit length, drought resistance, high temperature tolerance and YVMV resistance to be incorporated from the wild gene pool. Kuwada (1966) has developed an amphi-diploid of okra with Abelmoschus tuberculatus and described it as Abelmoschus $x$ tuberculatus. Further, $\mathrm{F}_{1}$ hybrids of $A$. tuberculatus with A. manihot were also attempted (Kuwada, 1974). Pal et al., (1952) attempted wide hybridization between cultivated okra and its closest progenitor, A. tuberculatus. Akhond et al., (2000) and Samarjeeva (2003) described the success of wide hybridization attempt involving okra with $A$. moschatus and $A$. angulosus, respectively.

Wide hybridization was attempted between various Abelmoschus species primarily for establishing the genetic relationship based on $F_{1}$ fertility and secondarily to derive useful new combinations (Joseph et al., 2013). The developed material could be of use for YVMV and Enation Leaf Curl Virus resistance apart from cytoplasmic male sterility in cultivated okra.

\section{Materials and Methods}

Ten taxa of Abelmoschus viz., A. angulosus var. angulosus, $A$. angulosus var. grandiflorus, A. caillei, A. esculentus, A. ficulneus, A. manihot var. tetraphyllus, A. moschatus ssp. moschatus, A. tetraphyllus var. pungens, A. tetraphyllus var. tetraphyllus and $A$. tuberculatus were raised in the vegetable block of ICAR-IIHR, Bengaluru during the kharif season of year 2015. Emasculation of mature flower buds was performed by removing the perianth and shaving off the anthers in the previous evening before anthesis and covered with butter paper cover. Ready to open flower buds were also covered at the same time with butter paper cover to collect pollen. Pollen grains from male parent were collected and dusted on the receptive stigmas of emasculated flowers between 8.00 AM and 10.30 AM and again covered with butter paper cover and tagged. Upon maturity, capsules were harvested, dried and hybrid seeds stored for 30-45 days in a cool place. Such seeds were physically scarified using sand-paper and soaked overnight in distilled water before sowing. Seedlings were raised in protrays. The 2-4 true leaf aged seedlings were transplanted to pots and allowed to grow under optimal conditions.

The list of wild species and cultivated genotypes used for crossability study was mentioned in Table 1.

\section{Results and Discussion}

Cross compatibility studies [explaining number of crosses attempted (NCA), number of fruit set (NFS), fruit set per cent (FSP), seed set per cent (SSP), crossability index per cent (CI \%)] between okra wild relatives and cultivated genotypes were mentioned in Table 2-14; among the wild relatives was mentioned in Table 15. The germination per cent of $F_{1}$ seeds and pollen viability (\%) of interspecific hybrids mentioned in Table 16.

There was no fruit set obtained when $A$. angulosus var. angulosus flowers pollinated with pollen from IIHR 294, IIHR 296, IIHR 299, IIHR 10-11-551, IIHR 10-11-875 and IIHR G 10. But good fruit set was noticed with IIHR 285(33.33\%) and with IIHR 10-11$594(69.23 \%)$. The highest crossability index percent $(38.10 \%)$ was recorded in the cross A. angulous var. angulosus X IIHR 285 followed by A. angulous var. angulosus X IIHR 594 (11.11\%) (Table 2). The highest crossability 
index per cent (15.24\%) was recorded in the cross A. angulosus var. grandiflorus IC 203832 X IIHR 294 followed by A. angulosus var. grandiflorus IC 203832 X IIHR 285 (Table 3). In the present investigation, there was no fruit set when $A$. angulosus var. grandiflorus-1 crossed with IIHR 294, IIHR 296, IIHR 299, IIHR 551, IIHR 875 and IIHR $\mathrm{G} 10$ whereas the appreciable fruit set was found only when $A$. angulosus var. grandiflorus-1 crossed with IIHR 285 and IIHR 594 (Table 4). But the seeds produced in these crosses were shriveled and non-viable. This suggests the existence of post-zygotic barriers during interspecific hybridization. The crosses between $A$. esculentus cultivars and $A$. angulosus were found successful by Prabu and Warade (2013) only when cultivated okra was used as female parent. When A.angulosus was used as the female parent incompatibility was observed resulting in failure of embryo formation by Samarajeewa et al., 1999. He opined that embryo rescue was the only alternative to raise viable hybrids. He found that when the cultivated type was used as female parent, progeny plants were produced without incompatibility difficulties.

The successful $\mathrm{F}_{1} \mathrm{~s}$ were obtained in both direct and reciprocal crosses between $A$. tetraphyllus var. tetraphyllus and $A$. esculentus cultivars by earlier workers [Jambhale (1980, Sheela (1986, 1994)]. The seed setting per cent was highest when crossed with IIHR 296 (100\%). But there was no fruit and seed set when $A$. tetraphyllus var. tetraphyllus crossed with A. esculentus lines IIHR 294, IIHR 299, IIHR 551 and IIHR 875. The present work is in conformity with previous findings in respect of successful crossing between A. tetraphyllus var. tetraphyllus and A. esculentus (Table 10) by Jambhale 1980. There was no fruit set and seed set observed when A. tetraphyllus var. pungense crossed with $A$. esculentus IIHR 285 and IIHR 10-11-594.
The results obtained in crosses between $A$. tuberculatus and A. escuelntus (Table 11) in the present study were in line with the findings of Kuwada (1966). The cross between $A$. tuberculatus and A. esculetnus genotype IIHR 10-11-594 recorded the highest fruit set (100\%) and seed set (99.02\%) and crossability index per cent $(100 \%)$ followed by $A$. tuberculatus X A. esculentus IIHR 10-11-875.

Abelmoschus ficulneus was found compatible when crossed to IIHR 285, IIHR 294, IIHR 299, IIHR 594 and G 10. The highest fruit set was found in the cross between A. ficulneus and IIHR 594 (69.23\%) followed by IIHR 285 $(21.88 \%)$ whereas the highest seed set was found in the cross between A. ficulneus and IIHR 294 (88.24\%) followed by G10 $(82.61 \%)$. The highest crossability index per cent was found in the cross between $A$. ficulneus and IIHR 594 (76.92\%) followed by $285(25.00 \%)$. But, A. ficulneus was found incompatible when $A$. esculentus line IIHR 296, IIHR 551 and IIHR 875 as pollen parents (Table 6). The main reason of hybrid inviability might be due to presence of irreconcilable parental chromosomes, negative cytoplasmic genic interactions and incongruous embryo and the surrounding tissue (Stebbins, 1958).

There was no fruit set when A. moschatus crossed with IIHR 294, IIHR 296, IIHR 299, IIHR 551, IIHR 875 and IIHR G 10 whereas the appreciable fruit set was found only when A. moschatus crossed with IIHR 285 and IIHR 594 (Table 10) in the present investigation.

But the seeds obtained were shriveled and non-viable. In this type of incompatibility, Allard (1990) opined that zygote formation might be prevented by ineffective growth of pollen or failure of fertilization. However, the viable hybrids of this species with $A$. esculentus through embryo culture technique were obtained by Gadwal et al., (1968). This 
indicates that A. moschatus is reproductively Hamon and Charrier (1983) and Pushaparajan less related to other okra species as opined by (1986).

Table.1 The list of wild species and cultivated genotypes used for crossability study

\begin{tabular}{|c|c|c|c|}
\hline $\begin{array}{l}\text { Sl. } \\
\text { No. }\end{array}$ & Wild species & $\begin{array}{l}\text { Sl. } \\
\text { No. }\end{array}$ & Cultivated species \\
\hline 1 & A. angulosus var. anglulosus & 1 & Abelmoschus esculentus IIHR 285 \\
\hline 2 & $\begin{array}{l}\text { A. angulosus var. grandiflorus IC } \\
203832\end{array}$ & 2 & Abelmoschus esculentus IIHR 294 \\
\hline 3 & A. angulosus var. grandiflorus -1 & 3 & Abelmoschus esculentus IIHR 296 \\
\hline 4 & A. caillei & 4 & Abelmoschus esculentus IIHR 299 \\
\hline 5 & A. ficulneus & 5 & $\begin{array}{l}\text { Abelmoschus esculentus IIHR 10-11- } \\
551\end{array}$ \\
\hline 6 & A. manihot var. tetraphyllus & 6 & $\begin{array}{l}\text { Abelmoschus esculentus IIHR 10-11- } \\
875\end{array}$ \\
\hline 7 & $\begin{array}{l}\text { A. manihot var. tetraphyllus IC } \\
203833\end{array}$ & 7 & $\begin{array}{l}\text { Abelmoschus esculentus IIHR 10-11- } \\
594\end{array}$ \\
\hline 8 & A. manihot var. tetraphyllus IC 90339 & 8 & Abelmoschus esculentus IIHR G 10 \\
\hline 9 & A. mizoramensis & & \\
\hline 10 & A. moschatus & & \\
\hline 11 & A. tetraphyllus var. pungense & & \\
\hline 12 & A. tetraphyllus var. tetraphyllus & & \\
\hline 13 & A. tuberculatus & & \\
\hline 14 & New taxa IC 0433556 & & \\
\hline
\end{tabular}


Table.2 Cross compatibility studies between A.angulosus var. angulosus and A. esculentus genotypes

\begin{tabular}{|c|c|c|c|c|c|c|c|}
\hline \multicolumn{3}{|c|}{ Cross combination } & NCA & NFS & FSP & SSP & CI \% \\
\hline A.angulosus var. angulosus & $X$ & IIHR 285 & 12 & 4 & 33.33 & 22.35 & 38.10 \\
\hline A.angulosus var. angulosus & $X$ & IIHR 294 & 10 & 0 & 0.00 & 0.00 & 0.00 \\
\hline A.angulosus var. angulosus & $\mathrm{X}$ & IIHR 296 & 10 & 0 & 0.00 & 0.00 & 0.00 \\
\hline A.angulosus var. angulosus & $\mathrm{X}$ & IIHR 299 & 10 & 0 & 0.00 & 0.00 & 0.00 \\
\hline A.angulosus var. angulosus & $\mathrm{X}$ & IIHR 10-11-551 & 10 & 0 & 0.00 & 0.00 & 0.00 \\
\hline A.angulosus var. angulosus & $\mathrm{X}$ & IIHR 10-11-594 & 10 & 1 & 10.00 & 69.23 & 11.11 \\
\hline A.angulosus var. angulosus & $\mathrm{X}$ & IIHR 10-11-875 & 15 & 0 & 0.00 & 0.00 & 0.00 \\
\hline A.angulosus var. angulosus & $\mathrm{X}$ & IIHR G 10 & 15 & 0 & 0.00 & 0.00 & 0.00 \\
\hline
\end{tabular}

Note: $\mathrm{NCA}=$ Number of Crosses Attempted, NFS $=$ Number of Fruit Set, FSP $=$ Fruit Set Per cent, SSP $=$ Seed Set Per cent $($ SSP), CI \% = Crossability Index Per cent

Table.3 Cross compatibility studies between A.angulosus var. grandiflorus IC 203832 and A. esculentus genotypes

\begin{tabular}{|c|c|c|c|c|c|c|c|}
\hline \multicolumn{3}{|c|}{ Cross combination } & NCA & NFS & FSP & SSP & CI \% \\
\hline A.angulosus var. grandiflorus IC 203832 & $\mathrm{X}$ & IIHR 285 & 15 & 2 & 13.33 & 42.31 & 14.81 \\
\hline A.angulosus var. grandiflorus IC 203832 & $\mathrm{X}$ & IIHR 294 & 15 & 2 & 13.33 & 36.36 & 15.24 \\
\hline A.angulosus var. grandiflorus IC 203832 & $\mathrm{X}$ & IIHR 296 & 10 & 0 & 0.00 & 0.00 & 0.00 \\
\hline A.angulosus var. grandiflorus IC 203832 & $\mathrm{X}$ & IIHR 299 & 10 & 0 & 0.00 & 0.00 & 0.00 \\
\hline A.angulosus var. grandiflorus IC 203832 & $X$ & IIHR 10-11-551 & 15 & 0 & 0.00 & 0.00 & 0.00 \\
\hline A.angulosus var. grandiflorus IC 203832 & $\mathrm{X}$ & IIHR 10-11-594 & 10 & 0 & 0.00 & 0.00 & 0.00 \\
\hline A.angulosus var. grandiflorus IC 203832 & $\mathrm{X}$ & IIHR 10-11-875 & 10 & 0 & 0.00 & 0.00 & 0.00 \\
\hline A.angulosus var. grandiflorus IC 203832 & $\mathrm{X}$ & IIHR G 10 & 10 & 0 & 0.00 & 0.00 & 0.00 \\
\hline
\end{tabular}


Table.4 Cross compatibility studies between A.angulosus var. grandiflorus-1 and A. esculentus genotypes

\begin{tabular}{|c|c|c|c|c|c|c|c|}
\hline \multicolumn{3}{|c|}{ Cross combination } & NCA & NFS & FSP & SSP & CI \% \\
\hline A.angulosus var. grandiflorus -1 & $\mathrm{X}$ & IIHR 285 & 81 & 14 & 17.28 & 48.33 & 19.75 \\
\hline A.angulosus var. grandiflorus -1 & $\mathrm{X}$ & IIHR 294 & 26 & 0 & 0.00 & 0.00 & 0.00 \\
\hline A.angulosus var. grandiflorus -1 & $\mathrm{X}$ & IIHR 296 & 30 & 0 & 0.00 & 97.30 & 0.00 \\
\hline A.angulosus var. grandiflorus -1 & $\mathrm{X}$ & IIHR 299 & 18 & 0 & 0.00 & 0.00 & 0.00 \\
\hline A.angulosus var. grandiflorus -1 & $\mathrm{X}$ & IIHR 10-11-551 & 10 & 0 & 0.00 & 0.00 & 0.00 \\
\hline A.angulosus var. grandiflorus -1 & $\mathrm{X}$ & IIHR 10-11-594 & 63 & 7 & 11.11 & 87.15 & 12.35 \\
\hline A.angulosus var. grandiflorus -1 & $\mathrm{X}$ & IIHR 10-11-875 & 10 & 0 & 0.00 & 0.00 & 0.00 \\
\hline A.angulosus var. grandiflorus -1 & $\mathrm{X}$ & IIHR G 10 & 10 & 0 & 0.00 & 0.00 & 0.00 \\
\hline
\end{tabular}

Table.5 Cross compatibility studies between A.caillei and A. esculentus genotypes

\begin{tabular}{|l|l|l|l|l|l|l|l|}
\hline \multicolumn{3}{|c|}{ Cross combination } & NCA & NFS & FSP & SSP & CI \% \\
\hline A. caillei & X & IIHR 285 & 19 & 9 & 47.37 & 99.60 & 48.58 \\
\hline A. caillei & X & IIHR 296 & 10 & 6 & 60.00 & 100.00 & 63.16 \\
\hline A. caillei & X & IIHR 299 & 10 & 8 & 80.00 & 100.00 & 84.21 \\
\hline A. caillei & X & IIHR 10-11-594 & 18 & 11 & 61.11 & 97.39 & 61.11 \\
\hline A. caillei & X & IIHR G 10 & 12 & 8 & 66.67 & 100.00 & 66.67 \\
\hline
\end{tabular}

Table.6 Cross compatibility studies between A. ficulneus and A. esculentus genotypes

\begin{tabular}{|l|l|l|l|l|l|l|l|}
\hline \multicolumn{2}{|c|}{ Cross combination } & NCA & NFS & FSP & SSP & CI \% \\
\hline A. ficulneus & X & IIHR 285 & 32 & 7 & 21.88 & 53.40 & 25.00 \\
\hline A. ficulneus & X & IIHR 294 & 13 & 1 & 7.69 & 88.24 & 9.05 \\
\hline A. ficulneus & X & IIHR 296 & 11 & 0 & 0.00 & 0.00 & 0.00 \\
\hline A. ficulneus & X & IIHR 299 & 12 & 1 & 8.33 & 50.00 & 9.80 \\
\hline A. ficulneus & X & IIHR 10-11-551 & 15 & 0 & 0.00 & 0.00 & 0.00 \\
\hline A. ficulneus & X & IIHR 10-11-594 & 13 & 9 & 69.23 & 64.53 & 76.92 \\
\hline A. ficulneus & X & IIHR 10-11-875 & 10 & 0 & 0.00 & 0.00 & 0.00 \\
\hline A. ficulneus & X & IIHR G 10 & 12 & 1 & 8.33 & 82.61 & 9.26 \\
\hline
\end{tabular}


Table.7 Cross compatibility studies between A manihot ssp. tetraphyllus and A. esculentus genotypes

\begin{tabular}{|c|c|c|c|c|c|c|c|}
\hline \multicolumn{3}{|c|}{ Cross combination } & NCA & NFS & FSP & SSP & CI \% \\
\hline A. manihot ssp. tetraphyllus & $X$ & IIHR 285 & 18 & 5 & 27.78 & 93.33 & 29.24 \\
\hline A. manihot ssp. tetraphyllus & $\mathrm{X}$ & IIHR 294 & 10 & 1 & 10.00 & 95.00 & 10.81 \\
\hline A. manihot ssp. tetraphyllus & $X$ & IIHR 296 & 10 & 3 & 30.00 & 94.74 & 32.43 \\
\hline A. manihot ssp. tetraphyllus & $\mathrm{X}$ & IIHR 299 & 10 & 1 & 10.00 & 66.67 & 10.81 \\
\hline A. manihot ssp. tetraphyllus & $\mathrm{X}$ & IIHR 10-11-551 & 10 & 2 & 20.00 & 93.94 & 21.62 \\
\hline A. manihot ssp. tetraphyllus & $\mathrm{X}$ & IIHR 10-11-594 & 15 & 2 & 13.33 & 92.31 & 13.68 \\
\hline A. manihot ssp. tetraphyllus & $X$ & IIHR 10-11-875 & 12 & 0 & 0.00 & 0.00 & 0.00 \\
\hline A. manihot ssp. tetraphyllus & $\mathrm{X}$ & IIHR G 10 & 15 & 1 & 6.67 & 64.00 & 6.84 \\
\hline
\end{tabular}

Table.8 Cross compatibility studies between A manihot ssp. tetraphyllus IC 203833 and A. esculentus genotypes

\begin{tabular}{|l|l|l|l|l|l|l|l|}
\hline \multicolumn{3}{|c|}{ Cross combination } & NCA & NFS & FSP & SSP & CI \% \\
\hline A. manihot ssp. tetraphyllus IC 203833 & X & IIHR 285 & 10 & 1 & 10.00 & 84.21 & 10.81 \\
\hline A. manihot ssp. tetraphyllus IC 203833 & X & IIHR 294 & 10 & 2 & 20.00 & 90.16 & 22.22 \\
\hline A. manihot ssp. tetraphyllus IC 203833 & X & IIHR 296 & 12 & 0 & 0.00 & 0.00 & 0.00 \\
\hline A. manihot ssp. tetraphyllus IC 203833 & X & IIHR 299 & 10 & 0 & 0.00 & 64.10 & 0.00 \\
\hline A. manihot ssp. tetraphyllus IC 203833 & X & IIHR 10-11-551 & 10 & 0 & 0.00 & 67.74 & 0.00 \\
\hline A. manihot ssp. tetraphyllus IC 203833 & X & IIHR 10-11-594 & 15 & 2 & 13.33 & 86.67 & 14.04 \\
\hline A. manihot ssp. tetraphyllus IC 203833 & X & IIHR 10-11-875 & 12 & 0 & 0.00 & 0.00 & 0.00 \\
\hline A. manihot ssp. tetraphyllus IC 203833 & X & IIHR G 10 & 10 & 0 & 0.00 & 0.00 & 0.00 \\
\hline
\end{tabular}


Table.9 Cross compatibility studies between A manihot ssp. tetraphyllus IC 90339 and A. esculentus genotypes

\begin{tabular}{|l|l|l|l|l|l|l|l|}
\hline \multicolumn{1}{|c|}{ Cross combination } & NCA & NFS & FSP & SSP & CI \% \\
\hline A. manihot ssp. tetraphyllus IC 90339 & X & IIHR 285 & 10 & 4 & 40.00 & 89.19 & 43.24 \\
\hline A. manihot ssp. tetraphyllus IC 90339 & X & IIHR 294 & 10 & 0 & 0.00 & 0.00 & 12.04 \\
\hline A. manihot ssp. tetraphyllus IC 90339 & X & IIHR 296 & 12 & 0 & 0.00 & 0.00 & 0.00 \\
\hline A. manihot ssp. tetraphyllus IC 90339 & X & IIHR 299 & 10 & 0 & 0.00 & 0.00 & 0.00 \\
\hline A. manihot ssp. tetraphyllus IC 90339 & X & IIHR 10-11-551 & 10 & 0 & 0.00 & 0.00 & 0.00 \\
\hline A. manihot ssp. tetraphyllus IC 90339 & X & IIHR 10-11-594 & 10 & 4 & 40.00 & 86.90 & 42.11 \\
\hline A. manihot ssp. tetraphyllus IC 90339 & X & IIHR 10-11-875 & 10 & 0 & 0.00 & 0.00 & 0.00 \\
\hline A. manihot ssp. tetraphyllus IC 90339 & X & IIHR G 10 & 12 & 0 & 0.00 & 0.00 & 0.00 \\
\hline
\end{tabular}

Table.10 Cross compatibility studies between A moschatus and A. esculentus genotypes

\begin{tabular}{|l|l|l|l|l|l|l|l|}
\hline \multicolumn{2}{|c|}{ Cross combination } & NCA & NFS & FSP & SSP & CI \% \\
\hline A. moschatus & X & IIHR 285 & 36 & 18 & 50.00 & 73.36 & 51.81 \\
\hline A. moschatus & X & IIHR 294 & 20 & 2 & 0.00 & 0.00 & 0.00 \\
\hline A. moschatus & X & IIHR 296 & 15 & 4 & 0.00 & 0.00 & 0.00 \\
\hline$A$. moschatus & X & IIHR 299 & 20 & 3 & 0.00 & 0.00 & 0.00 \\
\hline$A$. moschatus & X & IIHR 10-11-551 & 15 & 0 & 0.00 & 0.00 & 0.00 \\
\hline A. moschatus & X & IIHR 10-11-594 & 37 & 18 & 48.65 & 75.61 & 49.14 \\
\hline$A$. moschatus & X & IIHR 10-11-875 & 10 & 2 & 0.00 & 0.00 & 0.00 \\
\hline A. moschatus & X & IIHR G 10 & 15 & 4 & 0.00 & 0.00 & 0.00 \\
\hline
\end{tabular}

Table.11 Cross compatibility studies between A tetraphyllus var. pungense and A. esculentus genotypes

\begin{tabular}{|c|c|c|c|c|c|c|c|}
\hline \multicolumn{3}{|c|}{ Cross combination } & NCA & NFS & FSP & SSP & CI \% \\
\hline A. tetraphyllus var. pungense & $\mathrm{X}$ & IIHR 285 & 18 & 0 & 0.00 & 0.00 & 0.00 \\
\hline A. tetraphyllus var. pungense & $\mathrm{X}$ & IIHR 10-11-594 & 12 & 0 & 0.00 & 0.00 & 0.00 \\
\hline
\end{tabular}


Table.12 Cross compatibility studies between A tetraphyllus var. tetraphyllus and A. esculentus genotypes

\begin{tabular}{|c|c|c|c|c|c|c|c|}
\hline \multicolumn{3}{|c|}{ Cross combination } & NCA & NFS & FSP & SSP & CI \% \\
\hline A. tetraphyllus var. tetraphyllus & $\mathrm{X}$ & IIHR 285 & 32 & 17 & 53.13 & 94.23 & 57.43 \\
\hline A. tetraphyllus var. tetraphyllus & $\mathrm{X}$ & IIHR 294 & 20 & 0 & 0.00 & 0.00 & 0.00 \\
\hline A. tetraphyllus var. tetraphyllus & $\mathrm{X}$ & IIHR 296 & 15 & 1 & 6.67 & 100.00 & 7.41 \\
\hline A. tetraphyllus var. tetraphyllus & $\mathrm{X}$ & IIHR 299 & 20 & 0 & 0.00 & 0.00 & 0.00 \\
\hline A. tetraphyllus var. tetraphyllus & $\mathrm{X}$ & IIHR 10-11-551 & 15 & 0 & 0.00 & 0.00 & 0.00 \\
\hline A. tetraphyllus var. tetraphyllus & $\mathrm{X}$ & IIHR 10-11-594 & 36 & 16 & 44.44 & 95.73 & 46.78 \\
\hline A. tetraphyllus var. tetraphyllus & $\mathrm{X}$ & IIHR 10-11-875 & 10 & 0 & 0.00 & 0.00 & 0.00 \\
\hline A. tetraphyllus var. tetraphyllus & $\mathrm{X}$ & IIHR G 10 & 12 & 1 & 8.33 & 0.00 & 8.77 \\
\hline
\end{tabular}

Table.13 Cross compatibility studies between A tuberculatus and A. esculentus genotypes

\begin{tabular}{|c|c|c|c|c|c|c|c|}
\hline \multicolumn{3}{|c|}{ Cross combination } & NCA & NFS & FSP & SSP & CI \% \\
\hline A. tuberculatus & $\mathrm{X}$ & IIHR 285 & 16 & 7 & 43.75 & 98.20 & 44.87 \\
\hline A. tuberculatus & $\mathrm{X}$ & IIHR 294 & 10 & 3 & 30.00 & 97.03 & 31.58 \\
\hline A. tuberculatus & $\mathrm{X}$ & IIHR 296 & 10 & 0 & 0.00 & 0.00 & 0.00 \\
\hline A. tuberculatus & $\mathrm{X}$ & IIHR 299 & 10 & 3 & 30.00 & 100.00 & 31.58 \\
\hline A. tuberculatus & $\mathrm{X}$ & IIHR 10-11-551 & 10 & 2 & 20.00 & 100.00 & 21.05 \\
\hline A. tuberculatus & $\mathrm{X}$ & IIHR 10-11-594 & 10 & 10 & 100.00 & 99.02 & 100.00 \\
\hline A. tuberculatus & $\mathrm{X}$ & IIHR 10-11-875 & 10 & 5 & 50.00 & 100.00 & 51.28 \\
\hline A. tuberculatus & $X$ & IIHR G 10 & 10 & 3 & 30.00 & 86.57 & 30.00 \\
\hline
\end{tabular}

Table.14 Cross compatibility studies between New taxa IC 0433556 and A. esculentus genotypes

\begin{tabular}{|l|l|l|l|l|l|l|l|}
\hline \multicolumn{3}{|c|}{ Cross combination } & NCA & NFS & FSP & SSP & CI \% \\
\hline New taxa IC 0433556 & X & IIHR 285 & 15 & 0 & 0.00 & 0.00 & 0.00 \\
\hline New taxa IC 0433556 & X & IIHR 10-11-594 & 15 & 0 & 0.00 & 0.00 & 0.00 \\
\hline
\end{tabular}


Table.15 Cross compatibility studies between wild species of and A. esculentus genotypes

\begin{tabular}{|l|l|l|l|l|l|l|l|}
\hline \multicolumn{2}{|c|}{ Cross combination } & NCA & NFS & FSP & SSP & CI \% \\
\hline A. caillei & $\mathrm{X}$ & $\begin{array}{l}\text { A. angulosus var. } \\
\text { grandiflorus }\end{array}$ & 12 & 0 & 0.00 & 0.00 & 0.00 \\
\hline A. angulosus var. grandiflorus & $\mathrm{X}$ & $\begin{array}{l}\text { A. tetraphyllus ssp. } \\
\text { tetraphyllus }\end{array}$ & 9 & 0 & 0.00 & 0.00 & 0.00 \\
\hline A. angulosus var. grandiflorus & $\mathrm{X}$ & A. moshcatus & 14 & 0 & 0.00 & 0.00 & 0.00 \\
\hline A. moschatus & $\mathrm{X}$ & A. tuberculatus & 10 & 1 & 10.00 & 85.00 & 10.10 \\
\hline A. moschatus & $\mathrm{X}$ & $\begin{array}{l}\text { A. angulosus var. } \\
\text { grandiflorus }\end{array}$ & 12 & 2 & 16.67 & 75.00 & 18.73 \\
\hline A. angulosus var. grandiflorus & $\mathrm{X}$ & IIHR 10-11-875 & 10 & 0 & 0.00 & 0.00 & 0.00 \\
\hline A. angulosus var. grandiflorus & $\mathrm{X}$ & IIHR G 10 & 10 & 0 & 0.00 & 0.00 & 0.00 \\
\hline
\end{tabular}


In the present study, there was cross compatibility between $A$. caillei and $A$. esculentus (Table 5). The results are in conformity with the findings of Hamon and Hamon (1991). The highest fruit and seed set were observed when A. caillei was crossed with IIHR 299 (80.00\% and 100\%) followed by IIHR G 10 (66.67\% and 100\%). But, all the seeds obtained were not germinated due to lack of viable embryo. This may be resulted due to the differences in ploidy status of $A$. caillei as compared to A. esculentus which led to poor endosperm balance as per Kallo (1996). Though there was higher degree of crossability in the cross $A$. caillei $\mathrm{x} A$. esculentus, Chacko et al., (1996) noticed that the highest germination of the $\mathrm{F}_{1} \mathrm{~s}$ was observed only when $A$. esculentus was used as female parent. In contrast to this, Kousalya (2005) had reported maximum germination in the cross between A. caillei and A.esculentus.

The crosses attempted between A. manihot ssp. tetraphyllus IC 203833 and A. esculentus lines were successful when IIHR 285, IIHR 294 and IIHR 594 were used as the pollen parents but there was no fruit set when IIHR 296 and IIHR 299 used as the male parents (Table 8). The similar results were obtained when A. manihot ssp. tetraphyllus IC 90339 was used as the female parent (Table 9). Jambhale (1980) reported cross compatibility between A. manihot and A. esculentus but Teshima (1933) and Sujatha (1983) found that the cross was compatible only when $A$. esculentus was used as the female and $A$. manihot as the male parent.

From the present investigation, it was noted that higher values of fruit set per cent, number of seeds in crossed fruits, crossability index and germination per cent were recorded when A. esculentus lines used as the male parents while crossing with wild species viz., $A$. caillei, A. manihot ssp. tetraphyllus-1, A. manihot ssp. tetraphyllus-2 and $A$. tuberculatus. Whereas, the lowest values were recorded in crosses between $A$. angulosus var. grandiflorus -1, A. angulosus var. grandiflorus -2 and A. angulosus var. grandiflorus (IC 203832). This is in confirmation with Sheela (1994). The partial or complete failure of the endosperm to nurture the developing embryo due to the genetic imbalance might be the cause of the lowest seed set and recovery of shriveled seeds in the crossed fruits as observed by Allard, 1990. Whereas, Sindhu (1993) opined that lower fruit set was attributed to the chromosome number variation among the selected wild species. In the present study, seed germination was found maximum in crosses between cultivated okra species and Abelmoschus tetraphyllus. The obtained interspecific hybrids can be evaluated for identifying male sterile lines in the subsequent generations.

\section{Acknowledgement}

The author (Kattula Nagaraju) is thankful to UGC, New Delhi for the award of NFOBC during the research programme.

\section{References}

Akhond, Y.A.M, Molla, M.A.H., Islam, M.O., Ali, M. 2000. Cross compatibility between Abelmoschus esculentus and A. moschatus. Euphytica. 114 (3): 175 180.

Allard, R.W. 1990. In: Principles of plant breeding. John wiley and sons, New York- London. pp. 423-424.

Chacko, R.S., Sureshbabu, K.V., Rajan, S. 1996. Chromosome number of a semi wild form of okra. J. Trop. Agric. 34(1):138-139.

Dutta, O.P. 1976. Effects of gamma irradiation on seed germination, plant growth, flower biology and fruit production in okra (Abelmoschus 
esculentus (L.) Moench). Third Inter. Symposium on Trop. and Sub-tropical Hort. 1: 141-156.

Eshiet, A.J., Brisibe, E.A. 2015. Morphological Characterization and Yield Traits Analysis in Some Selected Varieties of Okra (Abelmoschus Esculentus L. Moench). Adv. Crop. Sci. Tech. 3:197.

Gadwal, V.R., Joshi, A.B., Iyer, R.D. 1968. Interspecific hybrids in Abelmoschus through ovule and embryo culture. Indian J. Genet. Plant Breed. 28:269274.

Gemede, H.F., Ratta, N., Haki, G.D., Woldegiorgis, A.Z. and Beyene, F. 2015. Nutritional quality and health benefits of okra (Abelmoschus esculentus): a review. J. Food Process Technol. 6: 458.

Gosslau, A., Chen, K.Y. 2004. Nutraceuticals, apoptosis, and disease prevention. Nutrition. 20: 95-102.

Hamon, S. and Charrier, A. 1983. Large variation of okra collected in Togo and Benin. Plant Genet. Resources- News letter. 56: 52-58.

Hamon, S. and Hamon, P. 1991. Future prospects of the genetic integrity to two species of okra (Abelmoschus esculentus and $A$. caillei) cultivated in West Africa, Euphytica. 58:101-111.

Jambhale, N.D. 1980. Cytogenetical studies in okra with reference to resistance to YVMV. Ph.D. Thesis, M.A.U., Parbhani.

John, J.K., Nissar, M.V.A., Latha, M., Patil, P., Malik, S.K., Negi, K.S., Keisham, M., Rao, S.R. Yadav, S.R. and Bhat, K.V. 2013. Genetic resources and crossability relationship among various species of Abelmoschus. Current Horticulture. 1(1): 35-46.

John, J.K., Roy, Y.C., Latha, M. and Bhat, K.V. 2017. In: Distant hybridization in Horticultural crops. Astral International
Pvt. Ltd., New Delhi. Pp. 155-165.

Kalloo, G. 1988. In: Distant hybridization in vegetable crop species. Vegetable breeding Vol. II. CRC press Inc. Florida, pp. 148-150.

Kousalya, V. 2005. Introgression of yellow vein mosaic resistance from $A$. caille $i$ (A. cher) Stevens into Abelmoschus esculentus (L.) Moench. M.Sc. (Agri.) Thesis, Kerala Agricultural University, Thrissur.

Kuwada, H. 1966. The new amphidiploid plant named Abelmoschus tubercularesculentus obtained from the progeny of the reciprocal crossing between $A$. tuberculatus and A. esculentus. Japanese J. Breed. 16: 21-30.

Kuwada, H. 1974. F 1 hybrids of Abelmoschus tuberculatus $\mathrm{X} A$. manihot with reference to the genome relationship. Jap. J. Breed. 24(5): 207-210.

Ohr, L.M. 2004. Dietary antioxidants. Food Technology. 58: 67-74.

Pal, B.P., Singh, H.B. and Swarup, V. 1952. Taxonomic relationships and breeding possibilities of species of Abelmoschus related to okra (A. esculentus). Bot. Gaz. 113:455-464.

Pitchaimuthu, M., Dutta, O.P. and Swamy, K.R.M. (2012). Studies on inheritance of geneic male sterility (GMS) and hybrid seed production in okra [Abelmoschus esculentus (L.) Moench.]. J. Hortl. Sci. 7(2): 199-202.

Prabu, T. and Warade, S.D. 2013. Crossability studies in genus Abelmoschus. Vegetable Science. 40(1): 11-16.

Pushparajan, G. 1986. Cytotaxonomic studies on South Indian Malvaceae, Ph.D. Thesis, Kerala Agricultural University, Thrissur. pp. 185.

Samarajeewa, P.K. 2003. Wild Abelmoschus species in the improvement of okra. In: Jayasuriya AHM and DA Vaughan (Ed.) Conservation and use of crop wild 
relatives. Proceedings of the Joint Department of Agriculture, Sri Lanka and National Institute of Agrobiological Sciences, Japan, workshop held on 302-2003 pp. 97-108.

Samarajeewa, P.K., Attanayake, P. and Gamage, N.S.T. 1999. Interspecific crosses between A.esculentus and A.angulosus. Tropical Agriculturist 152: 45-51.

Sheela, M. N. 1986. Evaluation of bhendi hybrids for yield and its components. M.Sc. (Agri.) Thesis, Kerala Agricultural University, Thrissur.

Sheela, M. N. 1994. Induction of genetic recombination in interspecific crosses of Abelmoschus. Ph.D. Thesis, Kerala Agricultural University, Thrissur.

Sindhu S. 1993. Interspecific cross compatibility in the genus Abelmoschus. M.Sc. (Agri.) Thesis, Kerala Agricultural University, Thrissur

Singh, B., Rai, M., Kalloo, G., Satpathy, S., Pandey K.K. 2007. Wild taxa of okra (Abelmoschus species): reservoir of genes for resistance to biotic stresses. In: Proc. $1^{\text {st }}$ IC on Indig. Veg. and Legumes. Eds. M.L. Chadha et al., Acta
Hort. 752, ISHS. Pp: 323-327.

Stebbins, G.L. 1958. The inviability, weakness and sterility of inter-specific hybrids. Adv. Genet., 9: 147-203.

Sujatha, V.S. 1983. Morphology of Abelmoschus spp. and crossability among them. M.Sc. (Agri.) Thesis, Indian Agricultural Research Institute, New Delhi.

Teshima T K.1933. Genetic and cytogenetical studies in an inter-specific hybrid of Hibiscus esculentus and H. manihot. J. Fac. Agri. Hokkaido. Univ. 34: 156.

Yamagishi, H. and Bhatt, S.R. (2014). Cytoplasmic male sterility in Brassicaceae crops. Breed Sci. 64(1): 38-47.

Yarrow S.A. (1993). Manipulation of cytoplasmic genomes. In: Labana, K.S., Banga, S.S., Banga, S.K. (Eds.), Breeding Oilseed Brassicas. Monographs on Theoretical and Applied Genetics, vol 19. (pp. 134144). Springer-Verlag Berlin Heidelberg New York. DoI: 10.1007/978-3-662-06166-4.

\section{How to cite this article:}

Kattula Nagaraju, M. Pitchaimuthu, A.T. Sadashiva, E.S. Rao, A. Rekha and Venugopalan, R. 2019. Interspecific Hybridization in Genus Abelmoschus Medikus. Int.J.Curr.Microbiol.App.Sci. 8(08): 425-438. doi: https://doi.org/10.20546/ijcmas.2019.808.048 